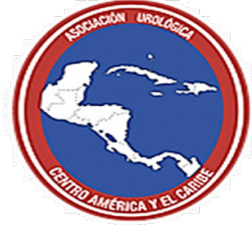

Asociación Urológica de Centroamérica y el Caribe

\section{URETROPLASTIA EN FASES CON INJERTO DE MUCOSA ORAL}

\section{Urethroplasty with Oral Mucosa Grafts in Stages}

Autores

Jenny María Rodríguez Cabrera ${ }^{(1)}$ \& Isis Emérita Pedro Silva ${ }^{(2)}$

(1) Hospital Clínico Quirúrgico Hermanos Ameijeiras, La Habana, Cuba.

Correo electrónico: jenny2011maria1988@gmail.com

DOI: https://doi.org/10.54212/27068048.v9i1.16

Esta investigación fue financiada con recursos propios. Declaramos no tener ningún conflicto de intereses

Fecha de envío: 26/04/2021

Fecha de aceptación: 03/06/2021

Fecha de publicación: 30/06/2021

\section{RESUMEN}

Introducción: La estrechez uretral es una de las principales causas de síntomas obstructivos en los hombres y con un impacto significativo en la vida por sus múltiples complicaciones. La uretroplastia en fases con injerto de mucosa oral ha devenido en una opción terapéutica para la estrechez uretral anterior compleja. Objetivo: Describir los resultados de la uretroplastia en fases con injerto de mucosa oral en la estrechez uretral anterior compleja. Metodología: Se realizó un estudio descriptivo, retrospectivo en 12 pacientes masculinos con estrechez uretral anterior compleja, en el Servicio de Urología del Hospital "Hermanos Ameijeiras", de Cuba, entre 2016-2020. Resultados: La edad media de la serie fue 41,7 años. Predominó el antecedente de hipospadias, la localización péndulo-bulbar y la estrechez entre $6,1-7 \mathrm{~cm}$. El 91,6\% tenían más de un tratamiento previo fallido, y la estenosis uretral era severa $(7,8)$. Predomino la re-estenosis con un $33,3 \%$, que fue solucionada en dos pacientes, para un éxito de $83,3 \%$. Los pacientes refirieron en un alto porcentaje mejoría de la calidad de vida y satisfacción con los resultados de la cirugía al completarse la segunda fase. Conclusiones: La uretroplastia en dos fases con injerto de mucosa oral es una opción terapéutica a considerar en la estrechez compleja de la uretra anterior por sus resultados satisfactorios.

\section{ABSTRACT}

Introduction: Urethral stricture is one of the main causes of obstructive symptoms in men with significant impact on their lives because of its multiple complications. Urethroplasty with oral mucosa grafts in stages has become a therapeutic option for complex anterior urethral stricture. Objective: To describe the results of urethroplasty with oral mucosa grafts in stages for complex anterior urethral stricture. Methodology: A descriptive, retrospective study of 12 male patients with complex anterior urethral stricture was carried out in the Service of Urology at the "Hermanos Ameijeiras" Hospital of Cuba, between 2016 and 2020. Results: The average age of the series was 41.7 years. Previous history of hypospadias, pendulum-bulbar location and stricture between 6.1 and $7 \mathrm{~cm}$ prevailed. $91.6 \%$ had more than one previous failed treatment, and severe urethral stenosis (7.8). Re-stenosis prevailed (33.3\%) which was resolved in two patients, $83.3 \%$ successfully. A high percentage of the patients reported that their quality of life was improved as well as being satisfied with the results of the surgery when the second stage was completed. Conclusions: Urethroplasty with oral mucosa grafts in two stages is a therapeutic option to be considered for complex anterior urethral stricture given its satisfactory results 


\section{PALABRAS CLAVE}

estrechez uretral, uretroplastia en fases, complicaciones

\section{INTRODUCCIÓN}

La estrechez uretral es una de las principales causas de síntomas obstructivos en los hombres con un significativo impacto por sus múltiples complicaciones. Su prevalencia en los países industrializados es aproximadamente $0,9 \%$. En Cuba, frecuentemente, es motivo de consulta lo que representa un problema de salud, así como, social y económico que repercute en todos los niveles de la vida del individuo y la familia, pero no se conocen datos exactos de su incidencia y prevalencia. (1) (2) La enfermedad por estrechez uretral puede desarrollarse a lo largo de toda la uretra masculina y su etiología puede ser idiopática, iatrogénica, por traumatismo externo, por infección, así como relacionada con el liquen escleroso (LE) y la hipospadia. La uretroplastia ofrece la tasa de éxito más alta y duradera con un $95 \%$ de éxito. La técnica varía ampliamente según la ubicación de la estenosis y su gravedad. (3) (4)

La mucosa de la cavidad bucal ha devenido en el injerto gold standard para la uretroplastia por ser un tejido preparado para la humedad, ser resistente a las infecciones, fácil de obtener y manipular. Actualmente, se ha descrito la uretroplastia por fases, que se reserva, principalmente, para casos complejos de reconstrucción uretral debido a la falta total de tejido sano con una vascularización precaria. Aunque los procedimientos en dos tiempos parecen soluciones de otra época, en que se asociaban a falta de pericia quirúrgica, en la actualidad ofrecen una solución en casos complejos de reconstrucción uretral. (5) $(6,7)(8)$

Se presenta la siguiente investigación con el objetivo de describir los resultados de la uretroplastia en fases con injerto de mucosa oral en la estrechez compleja de la uretra anterior.

\section{KEYWORDS}

urethral stricture, phased urethroplasty, complications

\section{METODOLOGÍA}

Se realizó un estudio observacional, descriptivo y retrospectivo en 12 pacientes masculinos con estrechez de uretra anterior, en el Servicio de Urología del Hospital Clínico Quirúrgico "Hermanos Ameijeiras", entre 2016-2020. Se incluyeron pacientes mayores de 18 años, con estrechez uretral compleja: tratamientos para estrechez uretral fallidos reiterados, asociados o no a hipospadia, LE, fistulas múltiples, pan-estrechez, espongiofibrosis, o longitud de la estrechez $\geq 4 \mathrm{cms}$. El procesamiento estadístico se realizó en un programa SPSS, versión 23.0. Se consideró un nivel de significación $p>0,05$. El estudio fue aprobado por el Comité de Ética Médica de la institución y se consideraron los principios de la Declaración de Helsinki. (9)

Técnica quirúrgica: Se realizó profilaxis antibiótica endovenosa con Cefazolina y Gentamicina. La cirugía se realizó bajo anestesia general. El paciente se colocó posición de litotomía. Se realizó incisión ventral longitudinal peneana, escrotal o perineal en dependencia de la localización y longitud de la estrechez. Para preparar el injerto de mucosa oral se realizó inyección submucosa en el carrillo con lidocaína al 0,9\% y se colocó en una solución preparada con dos ámpulas de gentamicina diluidas en $250 \mathrm{cc}$. En la primera fase se colocó el injerto a ambos lados de la incisión uretral en la cara dorsal y se suturó con Monocryl 4/0, previa extirpación de las áreas de espongiofibrosis (Fig 1). La uretra se dejó abierta y se colocó sonda uretro-vesical en el neo-meato proximal y si el paciente tenía cistotomía se conservaron ambos catéteres. Se empleó drenaje aspirativo y vendaje compresivo con solución antibiótica. La segunda fase (Fig. 2) se realizó, al menos, transcurridos seis meses de la primera, comprobando la ausencia 
de fibrosis y la permeabilidad del neo-meato proximal. Se confeccionó el tubo neo-uretral, que se delineó garantizando un ancho de placa de 2,5-3 cm, el cual se cerró intubado con un catéter uretral de silicona o látex $16 \mathrm{Fr}-18$

\section{RESULTADOS}

Predominaron los pacientes entre 30-39 años y el $91,6 \%$ eran menores de 60 años. Otras características de los pacientes de la serie se presentan en la Tabla 1.
Fr (Fig 3). Cierre de la piel (Fig 4). Se mantuvo vendaje compresivo durante $72 \mathrm{~h}$ y cateterismo uretro-vesical durante cuatro semanas.

Tabla 1. Características de los pacientes de la serie. UFMO. 2016-2020.

\begin{tabular}{|c|c|c|}
\hline Variables & Media & $\mathrm{DE}$ \\
\hline Edad & 41,7 & $\pm 12,81$ \\
\hline Longitud de la estenosis & $6,4 \mathrm{~cm}$ & $\pm 1,9 \mathrm{~cm}$ \\
\hline US score preoperatorio* & 7,8 & $\pm 1,1$ \\
\hline Complejidad de la estrechez(severa) $)^{* *}$ & 10 & 83,3 \\
\hline IPSS preoperatorio & & \\
\hline & No. & $\%$ \\
\hline Etiología hipospadias & 5 & 41,7 \\
\hline Etiología iatrogenia & 4 & 33,3 \\
\hline Localización péndulo bulbar & 9 & 75 \\
\hline Tratamientos previos $(+3)$ & 11 & 91,6 \\
\hline - $\quad$ Uretroplastia & 6 & 50 \\
\hline
\end{tabular}

*Urethral Stricture Score $\quad$ ** puntaje severo 8-9

En la primera fase la estenosis del neomeato fue la complicación más frecuente $(16,7 \%)$ y requirieron reintervención quirúrgica, después de la cual todos evolucionaron satisfactoriamente. En la segunda fase la re-estenosis $(33,3 \%)$ fue la complicación más frecuente, que apareció transcurrido 12 meses. Dos resolvieron con uretrotomía interna endoscópica (UIE) y dilatación uretral, respectivamente. 
Tabla 2. Complicaciones de ambas fases de la uretroplastia. UFMO. 2016-2020.

\begin{tabular}{|l|c|c|}
\hline \multirow{2}{*}{ Complicaciones } & 1ra Fase & 2da Fase \\
\cline { 2 - 3 } & No.l\% & No.I\% \\
\hline Pacientes complicados & $\mathbf{3}$ & $\mathbf{4}$ \\
\hline Estenosis del neomeato & $2 / 16,7$ & $2 / 16,7$ \\
\hline Infección urinaria & $1 / 8,3$ & $2 / 16,7$ \\
\hline Infección sitio operatorio & $1 / 8,3$ & $3 / 25$ \\
\hline Fistula uretral & & $4 / 33,3$ \\
\hline Re-estenosis uretral & & $1 / 8,3$ \\
\hline Curvatura peneana & - & \\
\hline
\end{tabular}

Los resultados de la uretroplastia en dos fases se muestran en la Tabla 3.

Tabla 3. Resultados de la uretroplastia en dos fases. UFMO. 2016-2020.

\begin{tabular}{|c|c|c|}
\hline Resultados & Media/Rango & DE \\
\hline Tiempo promedio entre fases (meses) & 13,6 & 7,3 \\
\hline \multirow[t]{2}{*}{ Media del IPSS* posoperatorio } & 1,1 & $\pm 2,4$ \\
\hline & No. & $\%$ \\
\hline Intervenciones adicionales primera fase & 3 & 25 \\
\hline Intervenciones adicionales segunda fase & 2 & 16,7 \\
\hline Re-estenosis al final del estudio & 2 & 16,7 \\
\hline Mejoría de calidad de vida USS-PROM ${ }^{* *}$ & 12 & 100 \\
\hline Satisfacción con la Cirugía & 10 & 83,3 \\
\hline Éxito & 10 & 83,3 \\
\hline
\end{tabular}

\section{DISCUSIÓN}

La mayor incidencia de la estrechez uretral ocurre en la 6ta década de la vida. Aunque se ha reportado una edad media de 49 y 50,1 años, otros investigadores reportan una edad menor (38,4 y 38,8 años). En los estudios sobre estrechez uretral cubanos la edad promedio ha sido similar al presente estudio. (1)(1013)(14)

Los países en desarrollo enfrentan principalmente la causa infecciosa los desarrollados el trauma. La causada por LE es la más desafiante. En otros la causa es desconocidas o por instrumentaciones. (15) (16)
La localización de la estrechez es un factor importante que repercute en el tratamiento y sus resultados. La estenosis del pene requiere de técnicas más complejas para su reconstrucción y el cuerpo esponjoso en esta zona es más delgado que en la porción bulbar. La incidencia de la estrechez en la uretra peneana fue mayor para unos autores, mientras para otros lo fue el segmento bulbopeneano.7) (16) (17)

Marco Spilotros y col (18) realizaron una clasificación de la longitud de las estenosis en $>4$ $\mathrm{cm}$, entre 4-8 cm y $<8 \mathrm{~cm}$ destacando menor recurrencia en relación con menor longitud. En numerosas series, a pesar de su elevada tasa de fracaso, el tratamiento inicial de la estenosis de uretra sigue siendo la UIE. Por 
cada centímetro que mida la estenosis se ha calculado que el riesgo relativo de fracaso de esta técnica aumenta un $22 \%$. Múltiples tratamientos endoscópicos previos predisponen a un abordaje abierto más complejo. (19)

La estenosis uretral es una complicación conocida después de la reparación de la hipospadia que conlleva a estrecheces más severas y tejido de mala calidad. $(20,21)$

El tratamiento de la estenosis uretral anterior compleja es un tema controversial y un continuo desafío, especialmente si es extensa o recurrente. En muchos casos la opción terapéutica es la uretroplastia en fases. (22) (7)

En situaciones tan complejas para la uretroplastia como las que se presentan en los pacientes de esta serie las complicaciones no son infrecuentes. Aunque en esta serie no se presentó, otros autores reportan sangrado en el sitio de la extracción de la mucosa y necesidad de suturas hemostáticas y entumecimiento oral, como complicaciones de la primera fase. La contractura del injerto que requiere reparación quirúrgica antes de completar la segunda fase ha sido reportada. Nathan y col. (23) en uretroplastia peneana en dos fases reportaron $36,0 \%$ de complicaciones. (18)

El tratamiento quirúrgico de la estrechez uretral con injerto de mucosa oral es efectivo siempre y cuando se tomen en cuenta los criterios de selección. (14). Se ha reportado tasas de éxito de $87,0 \%$ para la uretroplastia en dos fases VS $91 \%$ en solo tiempo y similares a lo reportado por otros. (23) (13) Mientras en el estudio múlticéntrico realizado por Barbagli G (18) la tasa de éxito fue $89,5 \%$ a los 44 meses. Para otros autores, el tiempo medio entre la primera y segunda fases fue 6,7 meses (rango: 6 - 9 meses. La literatura comenta que el $75 \%$ de la recurrencia de la estenosis uretral se observa dentro de los seis meses posteriores a la uretroplastia. La aplicación de la herramienta USS-PROM en los pacientes permitió mostrar de manera más objetiva la mejoría y su satisfacción ante la imposibilidad de aplicar la flujometría en esta investigación. Resulta- dos similares ha reportado otros autores con $84,3 \%$ de pacientes satisfechos o muy satisfechos, en un estudio donde se compararon medidas objetivas con subjetivas usando al paciente como su propio control lo que evidenció la confiabilidad del USS-PROM. (24) (25)

\section{CONCLUSIONES}

La uretroplastia en dos fases con injerto de mucosa oral es una opción terapéutica a considerar en la estrechez compleja de la uretra anterior por sus resultados satisfactorios.

\section{REFERENCIAS}

1. Smith TG, 3rd. Current management of urethral stricture disease. Indian $\mathrm{J}$ Urol. 2016;32(1):27-33.

https://doi.org/10.4103/0970-1591.173108

2. Palomeque Martínez M CPG, Navarro Cutiño M. Uretroplastia con mucosa prepucial en la estrechez uretral. Rev Cub Urol. 2018; Volumen 7.

3. Verla W, Oosterlinck W, Spinoit A-F, y cols. A Comprehensive review emphasizing anatomy, etiology, diagnosis, and treatment of male urethral stricture disease. Biomed Res Int. 2019. 9046430.

https://doi.org/10.1155/2019/9046430

4. Kałużny A, Krukowski J, Matuszewski M. Erectile dysfunction after urethroplasty. Cent European J Urol. 2019; 72(4):402-7.

https://doi.org/10.5173/ceju.2019.1737

5. Perdzyński W, Adamek M. Calibration of urethra with anti-scar gel as a new preventive method for stricture recurrence after urethroplasty performed due to refractory anterior urethral stricture disease. Cent European J Urol. 2020;73(1):80-90. 
6. Soave A, Kluth L, Dahlem R, y cols. Outcome of buccal mucosa graft urethroplasty: a detailed analysis of success, morbidity and quality of life in a contemporary patient cohort at a referral center. BMC Urol. 2019; 19: 18. https://doi.org/10.1186/s12894-019-0449-5

7. Selim M, Salem S, Elsherif E, y cols. Outcome of staged buccal mucosal graft for repair of long segment anterior urethral stricture. BMC Urol. 2019; 19:38.

https://doi.org/10.1186/s12894-019-0466-4

8. Gallo Rolanía F, S MA. Uretroplastias con cirugía en dos tiempos y uretrostomias perineales. Arch Esp Urol. 2011; 64 (10): 939-944.

9. General Assembly of the World Medical Association. World Medical Association Declaration of Helsinki: ethical principles for medical research involving human subjects. J Am Coll Dent. 2014 Summer; 81(3):14-8.

10. Nnabugwu II, Onuh AC, Anyimba SK, y cols. Comparing complications of urethral stricture across various ages: a retrospective analysis of findings from retrograde urethrogram and voiding cysto-urethrogram over 10 years. BMC Med Imaging. 2019; 19(84).

https://doi.org/10.1186/s12880-019-0384-7

11. Joshi P, Barbagli G, Batra V, y cols. A novel composite two-stage urethroplasty for complex penile strictures: A multicenter experience. Indian Journal of Urology. 2017; 33 (2): 155-158. https://doi.org/10.4103/0970-1591.203426

12.Şimşek A, Yenice MG, Şeker KG, y cols. Evaluation of the results of dorsolateral buccal mucosal augmentation urethroplasty. Turk J Urol. 2018; 45(3): 223-229.

https://doi.org/10.5152/tud.2018.47827

13. Wilson SC, Stranix JT, Khurana K, y cols. Fasciocutaneous flap reinforcement of ventral onlay buccal mucosa grafts enables neophallus revision urethroplasty. Ther Adv Urol. 2016; 8(6): 331-337.

https://doi.org/10.1177/1756287216673959
14.Pedro IE. Resultados de la uretroplastia con mucosa bucal en el tratamiento de la estrechez uretral bulbar. Rev Cub Urol. 2018; Volumen 7 .

15. Prabha V, Devaraju S, Vernekar R, y cols. Single stage: dorsolateral onlay buccal mucosal urethroplasty for long anterior urethral strictures using perineal route. Int Braz J Urol. 2016; 42(3): 564-570.

https://doi.org/10.1590/S1677-5538.IBJU.2015.0184

16. Selim M, Salem S, Elsherif E, y cols. Outcome of staged buccal mucosal graft for repair of long segment anterior urethral stricture. BMC Urol. 2019; 19(1):38.

https://doi.org/10.1186/s12894-019-0466-4

17. Alsagheer GA, Fathi A, Abdel-Kader MS, $y$ cols. Management of long segment anterior urethral stricture $(\geq 8 \mathrm{~cm})$ using buccal mucosal (BM) graft and penile skin (PS) flap: outcome and predictors of failure. Int Braz J Urol. 2018; 44(1): 163-171.

https://doi.org/10.1590/s1677-5538.ibju.2017.0083

18. Spilotros M, Sihra N, Malde S, y cols. Buccal mucosal graft urethroplasty in men-risk factors for recurrence and complications: a third referral centre experience in anterior urethroplasty using buccal mucosal graft. Transl Androl Urol. 2017 11-2019; 6(3):510-516.

https://doi.org/10.21037/tau.2017.03.69

19. Sukumar S, Elliott SP, Myers JB, y cols. Multi-Institutional Outcomes of Endoscopic Management of Stricture Recurrence after Bulbar Urethroplasty. J Urol. 2018; 200(4):837842. https://doi.org/10.1016/j.juro.2018.04.081

20. Jasionowska S, Brunckhorst O, Rees RW, y cols. Redo-urethroplasty for the management of recurrent urethral strictures in males: a systematic review. World J Urol. 2019; 37(9):1801-1815.

https://doi.org/10.1007/s00345-019-02709-7

21. Saavedra AA, Rourke KF. Characterization and outcomes of urethroplasty for hypospadias-associated urethral strictures in adults. 
Can Urol Assoc J. 2019; 13(11): E335-E40. https://doi.org/10.5489/cuaj.5863

22.Campos-Juanatey F, Bugeja S, Dragova $\mathrm{M}$, y cols. Single-stage tubular urethral reconstruction using oral grafts is an alternative to classical staged approach for selected penile urethral strictures. Asian J Androl. 2020; 22(2):134-139.

https://doi.org/10.4103/aja.aja_78_19

23. Hoy NY, Chapman DW, Rourke KF. Better defining the optimal management of penile urethral strictures: A retrospective comparison of single-stage vs. two-stage urethroplasty. Can Urol Assoc J. 2019; 13(12):414-418.

https://doi.org/10.5489/cuaj.5895
24. Ofoha CG, Ramyil VM, Dakum NK, y cols. Predictors of urethral stricture recurrence following urethroplasty: a retrospective review at the Jos University Teaching Hospital, Nigeria. Pan Afr Med J. 2019;32: 190.

https://doi.org/10.11604/pami.2019.32.190.18504

25. Lucas ET, Koff WJ, Rosito TE, y cols. Assessment of satisfaction and Quality of Life using self -reported questionnaires after urethroplasty: a prospective analysis. Int Braz J Urol. 2017; 43(2): 304-310.

https://doi.org/10.1590/s1677-5538.ibju.2016.0207

Copyright (c) 2021 Jenny María Rodríguez Cabrera y Isis Emérita Pedro Silva

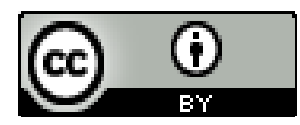

Este texto está protegido por una licencia Creative Commons 4.0.

Usted es libre para Compartir - copiar y redistribuir el material en cualquier medio o formato - y Adaptar el documento — remezclar, transformar y crear a partir del material— para cualquier propósito, , incluso para fines comerciales, siempre que cumpla la condición de:

Atribución: Usted debe dar crédito a la obra original de manera adecuada, proporcionar un enlace a la licencia, e indicar si se han realizado cambios. Puede hacerlo en cualquier forma razonable, pero no de forma tal que sugiera que tiene el apoyo del licenciante o lo recibe por el uso que hace de la obra. 